\title{
Adaptive Channel-Tracking Method and Equalization for MC-CDMA Systems over Rapidly Fading Channel under Colored Noise
}

\author{
Chang-Yi Yang ${ }^{1}$ and Bor-Sen Chen ${ }^{2}$ \\ ${ }^{1}$ Department of Computer Science and Information Engineering, National Penghu University, Penghu 88046, Taiwan \\ ${ }^{2}$ Department of Electrical Engineering, National Tsing-Hua University, Hsin-chu 300, Taiwan
}

Correspondence should be addressed to Chang-Yi Yang, cyyang@npu.edu.tw

Received 22 October 2009; Revised 30 June 2010; Accepted 13 July 2010

Academic Editor: Cihan Tepedelenlioğlu

Copyright (C) 2010 C.-Y. Yang and B.-S. Chen. This is an open access article distributed under the Creative Commons Attribution License, which permits unrestricted use, distribution, and reproduction in any medium, provided the original work is properly cited.

\begin{abstract}
A recursive maximum-likelihood (RML) algorithm for channel estimation under rapidly fading channel and colored noise in a multicarrier code-division multiple-access (MC-CDMA) system is proposed in this paper. A moving-average model with exogenous input (MAX) is given to describe the transmission channel and colored noise. Based on the pseudoregression method, the proposed RML algorithm can simultaneously estimate the parameters of channel and colored noise. Following the estimation results, these parameters can be used to enhance the minimum mean-square error (MMSE) equalizer. Considering high-speed mobile stations, a one-step linear trend predictor is added to improve symbol detection. Simulation results indicate that the proposed RML estimator can track the channel more precisely than the conventional estimator. Meanwhile, the performance of the proposed enhanced MMSE equalizer is robust to the rapidly Rayleigh fading channel under colored noise in the MC-CDMA systems.
\end{abstract}

\section{Introduction}

The direct-sequence code-division multiple-access (DSCDMA) technique has already been successfully implemented for third generation $(3 \mathrm{G})$ mobile communication systems [1-3]. Its utilization of channel bandwidth is efficient. Orthogonal frequency division multiplexing (OFDM) is a parallel transmission technique and has been adopted in IEEE802.16 [4, 5]. It can overcome the delay spread and spectrum efficiency in wireless communication systems. The idea of integrating the merits of both OFDM and CDMA schemes, known as multicarrier-CDMA (MC-CDMA), has attracted significant research interest recently. The MCCDMA system is a candidate technique for the next generation mobile communication system.

The MC-CDMA system divides the available bandwidth into a large number of narrow subchannels [6-8] and spreads each data symbol in the frequency domain by transmitting all the chips of a spread symbol at the same time, but in different orthogonal subchannels. One of the properties of multicarrier transmission is that the channel gain of each subchannel is different from the others. Since the MC-CDMA systems spread transmitted symbols in a nonflat fading channel, the inner product of different spreading codes will no longer be zero. This leads to the loss of orthogonality between different users. The multiple access interference (MAI) is introduced and the performance will be severely degraded in this situation. In order to preserve the orthogonality between different users, channel impairment should be estimated precisely and equalized efficiently. Earlier works [9-11] for MC-CDMA detectors made the assumption that the channel is perfectly known at the receiver. Recently, the impact of channel-estimation errors on the performance of MC-CDMA detectors has attracted much interest, and different approaches have been adopted for channel estimation and tracking. The pilotsymbol-aided channel-estimation methods in both time and frequency domains have been proposed [12-14], where the 
estimated channel coefficients are then obtained through the two-dimensional (2D) linear filtering. Other approaches $[15,16]$ consider an explicit channel estimation based on channel sounding in which a "train of pulses" spaced by the maximum delay spread of the channel is transmitted instead. A multiple channel model, which includes several possible channel models based on the different ranges of Doppler frequencies (or mobile velocities), is constructed to treat the time-varying fading channel [17]. In addition, a decisiondirected channel estimation in the frequency domain using Kalman-based filter has been proposed [18].

Although the MC-CDMA scheme is superior to noise and interference suppression, some papers $[19,20]$ have indicated that the narrowband interference (NBI) could affect its performance. The bandwidth of NBI does not exceed one subchannel. NBI is caused by intended jamming and some narrowband services. It can be considered as one kind of colored noise which has nonflat power spectral density and has been analyzed in $[21,22]$. NBI can be eliminated by notch filters in the CDMA systems [23-27]. The channel parameters are then estimated after despreading. But in the MC-CDMA systems, the objective of channel estimation is to make the gain of each subchannel equal for despreading. That is, the channel estimation and equalization should be done before despreading. Therefore, the notch filter will destruct the orthogonality and is not feasible for MCCDMA systems. The frequency-domain channel-estimation methods in the MC-CDMA systems will fail if the channel contains colored noise. It is because the colored noise can be considered as a white noise in each subchannel but with different power. When those pilot subcarriers suffer from noise with large power, the estimation results are no longer reliable and the performance degrades due to the loss of orthogonality between different users. Conventional estimation methods [28] are also unsuitable for channel estimation under colored noise because they will lead to a biased estimate due to the dependence between the residue and regression signal.

A channel-estimation algorithm for MC-CDMA systems under colored noise is proposed in this paper. It is derived based on the recursive maximum likelihood (RML) algorithm. It can jointly estimate the parameters of channel and colored noise in the time domain. A moving-average model with exogenous input (MAX) is used to describe the dynamics of channel and colored noise (or NBI). The colored noise is modeled as a moving-average (MA) process with a driving white noise. Since the driving white noise is unavailable, it is difficult to estimate the parameters of colored noise in the real time for precise channel estimation. The proposed method uses the estimated residue instead of the driving white noise to overcome this problem. After the channel parameters are estimated, they are fed to the minimum mean-square error (MMSE) detector to improve the performance of equalization.

The most important contribution of the proposed method is that the channel impulse response (CIR), the parameters of colored noise, and the power of the driving noise in the MC-CDMA systems can be simultaneously estimated in real time. Since the proposed method works in the time domain, it can significantly reduce the number of the estimated parameters compared with the methods in the frequency domain. For example, the number of the estimation algorithms in [18], which works in the frequency domain, should be 512 if there are 512 subchannels. Its computation algorithm is more complicated. But only an algorithm is needed for the channel estimation, no matter how many subchannels the MC-CDMA system has. However, the channel-parameter estimation in the time domain is easily deteriorated by colored noise. The proposed RML algorithm can overcome this problem with a simple scheme. Based on the estimation results, an enhanced MMSE equalizer is employed for symbol detection. The performance is demonstrated by the computer simulations.

This paper is organized as follows. In Section 2, the MCCDMA system is described. The proposed RML channel estimator is introduced in Section 3. The decision-directed channel-tracking process and the design of enhanced MMSE detector for the proposed method are presented in Section 4. The computer simulations of the adaptive MC-CDMA detector are presented and compared in Section 5, and the conclusions are summarized in Section 6. In what follows, $A^{T}$ denotes the transpose of $A$, and $A^{H}$ denotes the Hermitian of $A$. $\circledast$ denotes the circular convolution.

\section{MC-CDMA Communication System}

This section describes the models of the transmitter and receiver in an MC-CDMA system with $N_{s}$ subchannels and $N_{u}$ users. The $n$th multicarrier block symbol (duration $T$ ) for user $j$ is formed by taking $\mu$ symbols $d_{j}^{1}(n), \ldots, d_{j}^{\mu}(n)$ in parallel, which are spread by the user's spreading code $\mathbf{c}_{j}$ with length $N$. Thus, the $\mu$ spread symbols are placed into $N_{s}=$ $\mu N$ available subchannels. The transmitter's block diagram is shown in Figure 1. In the rest of this paper, for simplicity of notation, we concentrate only on the case where each user transmits one symbol $(\mu=1)$ in each MC-CDMA block symbol. The transmitted symbol is simply represented as $d_{j}(n)$ for user $j$. Therefore, the total number of subchannels is $N_{s}=N$.

2.1. Model of Transmitter. The Walsh-Hadamard spread code $\mathbf{c}_{j}$ for user $j$ with length $N$ is

$$
\mathbf{c}_{j}=\left[\begin{array}{llll}
c_{j}(0) & c_{j}(1) & \cdots & c_{j}(N-1)
\end{array}\right]^{T},
$$

where $c_{j}(n) \in\{1 / \sqrt{N},-1 / \sqrt{\mathrm{N}}\}$ and

$$
\mathbf{c}_{i}^{T} \mathbf{c}_{j}= \begin{cases}1, & \text { if } i=j, \\ 0, & \text { otherwise. }\end{cases}
$$

The $n$th symbols with spread sequence of all $N_{u}$ users transmitted on the $m$ th subchannel can be written as

$$
S_{n}(m)=\sum_{j=1}^{N_{u}} c_{j}(m) d_{j}(n)
$$






FIGURE 1: Block diagram of the MC-CDMA transmitter.

for $m=0,1, \ldots, N-1$. The signal $S_{n}(m)$ is then translated by the multicarrier modulation (i.e., IDFT), and the OFDM symbol $s_{n}(k)$ is produced as follows (see Figure 1):

$$
s_{n}(k)=\frac{1}{\sqrt{N}} \sum_{m=0}^{N-1} S_{n}(m) e^{j 2 \pi m k / N}
$$

for $k=0,1, \ldots, N-1$. A guard interval is inserted between successive OFDM symbols to avoid the ISI effects by using a cyclic prefix technique [29]. After the parallel-to-serial conversion, the combination of the cyclic prefix with the IDFT output sequence is given by

$$
s_{n}^{g}(k)=s_{n}(k+N-G) \bmod N,
$$

for $k=0,1, \ldots, N+G-1$, where the subscript $n$ and superscript $g$ denote the $n$th signal block with a guard interval. Thus, the total MC-CDMA symbol duration is $(N+$ $G) T_{c}$, where $G T_{c}$ is the guard interval duration and $T_{c}$ is the sampling time. The value of $G T_{c}$ is larger or equal to the maximum multipath delay spread $\tau_{\max }$ of the channel, and is small in respect to $T_{s}=N T_{c}$ (a useful OFDM symbol duration) to limit the spectral efficiency loss.

2.2. Model of Receiver. In the downlink case, all users' transmitting signals are synchronous and experience the same Rayleigh fading channel. The received signal of the $n$th MC-CDMA symbol is given by

$$
y_{n}^{g}(k)=\sum_{l=0}^{L-1} s_{n}^{g}(k-l) h_{n}(k, l)+v_{n}^{g}(k)
$$

for $k=0,1, \ldots, N+G-1$, where $h_{n}(k, l)$ and $v_{n}^{g}(k)$ denote the $l$ th path sample of the complex time-varying fading channel with length $L$ and the additive colored noise at the $k$ th instant of the $n$th MC-CDMA block symbol, respectively. For a high data-rate transmission, it is reasonably assumed that the channel is time invariant during one MC-CDMA block symbol interval $T$ [18], that is, $h_{n}(0, l)=h_{n}(1, l)=$ $\cdots=h_{n}(N+G-1, l)$ for $l=0,1, \ldots, L-1$. The index $k$ of $h_{n}(k, l)$ would be ignored in this case and can be simply rewritten as $h_{n}(l)$. However, channel variation during the successive symbol intervals is allowed. After removing the guard interval from (6), the received signal can be described as

$$
y_{n}(k)=y_{n}^{g}(k+G)=s_{n}(k) \circledast h_{n}(k)+v_{n}(k)
$$

for $k=0,1, \ldots, N-1$. Since $v_{n}(k)$ is a colored noise, it can be modeled by an MA process

$$
v_{n}(k)=u_{n}(k)+\sum_{p=1}^{P} a_{n}(p) u_{n}(k-p)
$$

where $u_{n}(k)$ is a driving noise modeled as zero-mean white-Gaussian process with variance $\sigma_{u}^{2}$, and $a_{n}(p)$ is the coefficient of the $p$ th tap at the $n$th symbol period. The parameters $a_{n}(p)$ can be set to 0 for $p=1, \ldots, P$ if the 
additive noise is white. After passing through DFT, the received signal on the $m$ th subchannel $Y_{n}(m)$ is

$$
\begin{aligned}
Y_{n}(m) & =\frac{1}{\sqrt{N}} \sum_{k=0}^{N-1} y_{n}(k) e^{-j 2 \pi k m / N} \\
& =\frac{1}{\sqrt{N}} \sum_{k=0}^{N-1}\left[s_{n}(k) \circledast h_{n}(k)+u_{n}(k) \circledast a_{n}(k)\right] e^{-j 2 \pi k m / N} \\
& =S_{n}(m) H_{n}(m)+U_{n}(m) A_{n}(m),
\end{aligned}
$$

where

$$
\begin{aligned}
& H_{n}(m)=\sum_{l=0}^{N-1} h_{n}(l) e^{-j 2 \pi l m / N}, \\
& A_{n}(m)=\sum_{p=0}^{N-1} a_{n}(p) e^{-j 2 \pi p m / N},
\end{aligned}
$$

$a_{n}(0)=1$, and $U_{n}(m)$ is the frequency-domain noise. The covariance of $U_{n}(m)$ can be expressed as

$$
\begin{aligned}
Q_{U_{n}}(m) & =E\left\{U_{n}(m) U_{n}^{H}(m)\right\} \\
& =N \sigma_{u}^{2} .
\end{aligned}
$$

Because the term $A_{n}(m)$, due to colored noise, may be large at some $n$ and $m$, the frequency-domain pilot-aided channel estimation for $H_{n}(m)$ is not reliable if the interference power $A_{n}(m)$ is high at that subchannel. Therefore, it is not easy to estimate channel $H_{n}(m)$ accurately from the received signal $Y_{n}(m)$ in the frequency domain. Furthermore, if the number of subchannels is large, many parameters need to be estimated. An RML method is proposed to jointly estimate the coefficients of channel and colored noise in (7) and (8) by the time-domain method via a pseudoregression scheme in the next section. An MMSE detector is designed based on the estimated coefficients to compensate the effects of the colored noise.

\section{RML Channel Estimation in the Training Mode}

The proposed RML channel estimator is developed to eliminate the effect of colored noise in this section. It works in the time domain, that is, the channel is estimated before
DFT. Combining (7) and (8), the receiving signal $y_{n}(k)$ can be expressed as

$$
\begin{aligned}
y_{n}(k)= & \sum_{l=0}^{L-1} h_{n}(l) s_{n}(k-l) \\
& +\sum_{p=1}^{P} a_{n}(l) u_{n}(k-l)+u_{n}(k) \\
= & {\left[\begin{array}{llll}
s_{n}(k) & s_{n}(k-1) & \cdots & s_{n}(k-L+1)
\end{array}\right] } \\
& +\left[\begin{array}{lll}
u_{n}(k-1) & \cdots & u_{n}(k-P)
\end{array}\right]\left[\begin{array}{c}
a_{n}(1) \\
\vdots \\
a_{n}(P)
\end{array}\right] \\
& +u_{n}(k) \\
= & \mathbf{s}_{k, n}^{T} \mathbf{h}_{n}+\mathbf{u}_{k, n}^{T} \mathbf{a}_{n}+u_{n}(k) \\
= & \phi_{k, n}^{H} \boldsymbol{\theta}_{n}+u_{n}(k),
\end{aligned}
$$$$
=\left[\begin{array}{llll}
s_{n}(k) & s_{n}(k-1) & \cdots & s_{n}(k-L+1)
\end{array}\right]\left[\begin{array}{c}
h_{n}(0) \\
h_{n}(1) \\
\vdots \\
h_{n}(L-1)
\end{array}\right]
$$

where

$$
\begin{aligned}
& \mathbf{s}_{k, n}=\left[\begin{array}{llll}
s_{n}(k) & s_{n}(k-1) & \cdots & s_{n}(k-L+1)
\end{array}\right]^{T}, \\
& \mathbf{h}_{n}=\left[\begin{array}{llll}
h_{n}(0) & h_{n}(1) & \cdots & h_{n}(L-1)
\end{array}\right]^{T}, \\
& \mathbf{u}_{k, n}=\left[\begin{array}{llll}
u_{n}(k-1) & u_{n}(k-2) & \cdots & u_{n}(k-P)
\end{array}\right]^{T}, \\
& \mathbf{a}_{n}=\left[\begin{array}{llll}
a_{n}(1) & a_{n}(2) & \cdots & a_{n}(P)
\end{array}\right]^{T}, \\
& \phi_{k, n}=\left[\begin{array}{ll}
\mathbf{s}_{k, n}^{T} & \mathbf{u}_{k, n}^{T}
\end{array}\right]^{H}, \\
& \boldsymbol{\theta}_{n}=\left[\begin{array}{ll}
\mathbf{h}_{n}^{T} & \mathbf{a}_{n}^{T}
\end{array}\right]^{T} \\
& a_{n}(0)=1 \text {. }
\end{aligned}
$$

The description of the transmission system in (12) is called an MAX model. It is a simple form of ARMAX model [30]. Since the driving noise $u_{n}(k)$ is white, the covariance matrix of the driving noise $\mathbf{u}_{n}$ is $\Sigma_{u_{n}}=E\left[\mathbf{u}_{n} \mathbf{u}_{n}^{H}\right]=$ $\sigma_{u}^{2} I$ and assumed to be unknown at the receiver, where $\mathbf{u}_{n}=\left[\begin{array}{lll}u_{n}(0) & \cdots & u_{n}(N-1)\end{array}\right]^{T}$. The parameters $\theta$ should be estimated in the colored noise case to obtain accurate channel model, that is, the parameters of the channel model and colored noise will be simultaneously estimated from (12). However, the regression vector $\phi_{k}$ contains noises $u_{n}(k-1) \quad \cdots u_{n}(k-P)$, which are unavailable and should be replaced by pseudoregression vector in the sequel. 
Formulation of a maximum-likelihood channel-estimation problem involves the derivation of a likelihood function $L(\boldsymbol{\theta})$,

$$
L_{n}(\boldsymbol{\theta})=\frac{1}{(2 \pi)^{N / 2}\left(\operatorname{det} \Sigma_{u_{n}}\right)^{1 / 2}} e^{-(1 / 2) \varepsilon_{n}^{H}(\boldsymbol{\theta}) \Sigma_{u_{\mathrm{n}}}^{-1} \varepsilon_{n}(\boldsymbol{\theta})}
$$

or the log-likelihood function

$$
\log L_{n}(\boldsymbol{\theta})=-\frac{1}{2} \log \left[(2 \pi)^{N} \operatorname{det} \Sigma_{u_{n}}\right]-\frac{1}{2} \varepsilon_{n}^{H}(\boldsymbol{\theta}) \Sigma_{u_{n}}^{-1} \varepsilon_{n}(\boldsymbol{\theta}),
$$

where

$$
\begin{aligned}
\mathcal{\varepsilon}_{n}(\boldsymbol{\theta}) & =\left[\begin{array}{llll}
\varepsilon_{0, n}(\boldsymbol{\theta}) & \varepsilon_{1, n}(\boldsymbol{\theta}) & \cdots & \varepsilon_{N-1, n}(\boldsymbol{\theta})
\end{array}\right]^{T}, \\
\mathcal{\varepsilon}_{k, n}(\boldsymbol{\theta}) & =y_{n}(k)-\phi_{k, n}^{H} \boldsymbol{\theta}_{n} .
\end{aligned}
$$

Since the noise $u_{n}(k)$ is assumed as a zero-mean whiteGaussian process and its variance $\sigma_{u}^{2}$ is unknown, (15) will be replaced by the following log-likelihood function:

$$
\begin{aligned}
\log L_{n}\left(\boldsymbol{\theta}, \sigma_{u}^{2}\right) & =-\frac{N}{2} \log (2 \pi)-\frac{N}{2} \log \sigma_{u}^{2}-\frac{1}{2 \sigma_{u}^{2}} \sum_{k=0}^{N-1}\left|\varepsilon_{k, n}(\boldsymbol{\theta})\right|^{2} \\
& =-\frac{N}{2} \log (2 \pi)-\frac{N}{2} \log \sigma_{u}^{2}-\frac{1}{\sigma_{u}^{2}} V_{N, n}(\boldsymbol{\theta}),
\end{aligned}
$$

where

$$
\begin{aligned}
V_{N, n}(\boldsymbol{\theta}) & =\frac{1}{2} \sum_{k=0}^{N-1}\left|\varepsilon_{k, n}(\boldsymbol{\theta})\right|^{2} \\
& =\frac{1}{2} \varepsilon_{n}^{H}(\boldsymbol{\theta}) \varepsilon_{n}(\boldsymbol{\theta}) \\
& =\frac{1}{2}\left(\mathbf{y}_{n}-\Phi_{N, n}^{H} \boldsymbol{\theta}_{n}\right)^{H}\left(\mathbf{y}_{n}-\Phi_{N, n}^{H} \boldsymbol{\theta}_{n}\right), \\
\Phi_{N, n} & =\left[\begin{array}{llll}
\phi_{0, n} & \phi_{1, n} & \cdots & \phi_{N-1, n}
\end{array}\right], \\
\mathbf{y}_{n} & =\left[\begin{array}{llll}
y_{n}(0) & y_{n}(1) & \cdots & y_{n}(N-1)
\end{array}\right]^{T} .
\end{aligned}
$$

The maximum-likelihood parameter estimation is to specify $\boldsymbol{\theta}$ and $\sigma_{u}^{2}$ to maximize the log-likelihood function in (17). Using the least-square (LS) criteria, we get the following estimation [28]:

$$
\begin{aligned}
\hat{\boldsymbol{\theta}}_{n} & =\left(\Phi_{N, n} \Phi_{N, n}^{H}\right)^{-1} \Phi_{N, n} \mathbf{y}_{n}, \\
\hat{\sigma}_{u}^{2} & =\frac{2}{N} V_{N, n}\left(\hat{\boldsymbol{\theta}}_{n}\right) .
\end{aligned}
$$

Therefore, the maximum-likelihood parameter estimation can be obtained. But there are still two problems. One is that the noise components of $\phi_{k}$, that is, $u_{n}(k-1), \ldots, u_{n}(k-P)$, are unavailable. The other is that the parameter estimation in (19) is in block form, which is not suitable for realtime design in the MC-CDMA communication systems. A recursive parameter estimation for (19) and an estimate for noise sequence $u_{n}(k-1), \ldots, u_{n}(k-P)$ are necessary in this situation.

The recursive maximum likelihood (RML) method is proposed via pseudolinear regression method in the following equation [28]:

$$
\begin{gathered}
\hat{\boldsymbol{\theta}}_{k, n}=\hat{\boldsymbol{\theta}}_{k-1, n}+\frac{P_{k-1, n} \phi_{k, n}}{\lambda+\phi_{k, n}^{H} P_{k-1, n} \phi_{k, n}}\left[y_{n}(k)-\phi_{k, n}^{H} \hat{\boldsymbol{\theta}}_{k-1, n}\right], \\
P_{k, n}=\frac{1}{\lambda}\left(P_{k-1, n}-\frac{P_{k-1, n} \phi_{k, n} \phi_{k, n}^{H} P_{k-1, n}}{\lambda+\phi_{k, n}^{H} P_{k-1, n} \phi_{k, n}}\right)
\end{gathered}
$$

which is the modified formulation of recursive least square (RLS) method with a forgetting factor $\lambda$ for improving the rate of convergence in the time-varying channel. Originally, the regression vector $\phi_{k}$ used for recursive estimation is

$$
\begin{aligned}
& {\left[\begin{array}{llll}
s_{n}(k) & s_{n}(k-1) & \cdots & s_{n}(k-L+1)
\end{array}\right.} \\
& \left.u_{n}(k-1) \quad u_{n}(k-2) \quad \cdots \quad u_{n}(k-P)\right]^{H} .
\end{aligned}
$$

It, of course, cannot be implemented since the driving noise $\mathbf{u}_{k, n}$ is unavailable by measurement. From (12), we get $u_{n}(k)=y_{n}(k)-\phi_{k, n}^{H} \boldsymbol{\theta}_{n}$. Therefore, the residue $\epsilon_{k, n}$,

$$
\epsilon_{k, n}=y_{n}(k)-\phi_{k, n}^{H} \widehat{\boldsymbol{\theta}}_{k, n},
$$

can be an estimate of $u_{n}(k)$. That means the noise components of $\phi_{k}$ can be substituted by their residues. The regression vector $\phi_{k, n}$ should be modified to the following at each recursive step in (20),

$$
\begin{gathered}
\phi_{k, n}=\left[\begin{array}{cccc}
s_{n}(k) & s_{n}(k-1) & \cdots & s_{n}(k-L+1) \\
\epsilon_{k-1, n} & \epsilon_{k-2, n} & \cdots & \epsilon_{k-P, n}
\end{array}\right]^{H} .
\end{gathered}
$$

The vector in (23) is called the pseudoregression vector because noise component of the desired regression vector is replaced by the estimated residues. The proposed RML estimation algorithm is in the form of RLS algorithm but is more powerful than the conventional RLS in the colored noise case. Finally, the estimate of the variance of driving noise is derived by using the residues in each recursion:

$$
\hat{\sigma}_{u}^{2}=\frac{1}{N} \sum_{k=0}^{N-1}\left|\epsilon_{k, n}\right|^{2}
$$

\section{Adaptive Channel Tracking and Prediction in the Tracking Mode}

Since the wireless mobile channel is time-varying, the channel model must be tracked continuously at the receiver for the correct data detection. The proposed RML algorithm in the previous section assumes the transmitted signal $s_{n}(k)$ can be obtained. This is only true in the training mode, but the transmitted signal is unknown for receiver in the tracking mode. Therefore, the detected data will be fed to the RML estimator to replace the transmitted signal for channel estimation. This is the so-called decision-directed channel estimation. 
4.1. Decision-Directed Algorithm in the Tracking Mode. In this subsection, an adaptive decision-directed channelestimation algorithm in the time domain is developed. The conventional channel-estimation methods for multicarrier transmission systems are equipped with pilots in the frequency domain, which is spaced by coherence bandwidth. When colored noise or NBI exists, the noise can be considered as white noise but with different powers in each subchannel after DFT conversion at the receiver. As a result, the pilot-symbol-aided channel-estimation methods $[12,13]$ are no longer reliable because some pilot subcarriers suffer from white noise with large power. Another drawback of these methods is the occupancy of bandwidth, which is a valuable resource for the service providers. Decision-directed channel tracking is a solution to this problem since it does not need any bandwidth and has a good performance in the well-known channel [18]. But the parameters of channel and colored noise are usually time varying. Therefore, the proposed RML method is modified here for channel tracking.

The data-flow diagram of the proposed decision-directed algorithm is shown in Figure 2. The detected symbol $\hat{\mathbf{d}}_{n}$ is used instead of the transmitted signal $\mathbf{d}_{n}$ to identify the channel (since the MMSE data detection scheme is introduced in Section 4.3, it is assumed in this subsection that $\widehat{\mathbf{d}}_{n}$ is available). Therefore, the parameters related to $\mathbf{d}_{n}$ in the RML algorithm should be regenerated from $\hat{\mathbf{d}}_{n}$ :

$$
\hat{s}_{n}(k)=\frac{1}{\sqrt{N}} \sum_{m=0}^{N-1} \sum_{j=1}^{N_{u}} c_{j}(m) \hat{d}_{j}(n) e^{j 2 \pi m k / N} .
$$

Thus, the regression vector in the channel-parametertracking method is modified as

$$
\begin{gathered}
\hat{\phi}_{k, n}=\left[\begin{array}{cccc}
\hat{s}_{n}(k) & \hat{s}_{n}(k-1) & \cdots & \hat{s}_{n}(k-L+1) \\
\epsilon_{k-1, n} & \epsilon_{k-2, n} & \cdots & \epsilon_{k-P, n}
\end{array}\right]^{H} .
\end{gathered}
$$

Based on the RML algorithm in (20), the following channelparameter-tracking scheme is proposed:

$$
\begin{aligned}
\hat{\boldsymbol{\theta}}_{k, n} & =\hat{\boldsymbol{\theta}}_{k-1, n}+\frac{P_{k-1, n} \hat{\phi}_{k, n}}{\lambda+\hat{\phi}_{k, n}^{H} P_{k-1, n} \hat{\phi}_{k, n}}\left[y_{n}(k)-\hat{\phi}_{k, n}^{H} \hat{\boldsymbol{\theta}}_{k-1, n}\right], \\
P_{k, n} & =\frac{1}{\lambda}\left(P_{k-1, n}-\frac{P_{k-1, n} \hat{\phi}_{k, n} \hat{\phi}_{k, n}^{H} P_{k-1, n}}{\lambda+\hat{\phi}_{k, n}^{H} P_{k-1, n} \hat{\phi}_{k, n}}\right), \\
\epsilon_{k, n} & =y_{n}(k)-\hat{\phi}_{k, n}^{H} \hat{\boldsymbol{\theta}}_{k, n},
\end{aligned}
$$

and the variance of driving noise is calculated as

$$
\hat{\sigma}_{u}^{2}=\frac{1}{N} \sum_{k=0}^{N-1}\left|\epsilon_{k, n}\right|^{2} .
$$

The initial condition $\hat{\boldsymbol{\theta}}_{0, n}$ is equal to $\hat{\boldsymbol{\theta}}_{N, n-1}$ which is the latest channel estimation of the previous MC-CDMA symbol and $N$ is the number of the recursion in one symbol duration. In what follows, $\hat{\boldsymbol{\theta}}_{n}$ is equal to $\hat{\boldsymbol{\theta}}_{N, n-1}$.
Since the transmitted signal $s_{n}(k)$ is unavailable for receiver in the tracking mode, the regenerated signal $\hat{s}_{n}(k)$ from the detected data $\hat{\mathbf{d}}_{n}$ in (25) is directly replaced for parameter tracking. In this way, the current channelparameter tracking is done after the current data detection. However, the MMSE data detection needs to have the current channel parameters. This is a delay problem of the decisiondirected channel-tracking scheme. A conventional decisiondirected scheme [18] adopts the previous estimates $\hat{\boldsymbol{\theta}}_{n-1}$ for the current MMSE data detection $\hat{\mathbf{d}}_{n}$, based on the assumption that the channel variation is slow. However, when the channel variation is fast, the previous estimates will not be suitable for the current data detection. In order to design MMSE detector at time $n$, the parameter $\hat{\boldsymbol{\theta}}_{n}$ must be predicted from the previous parameter-estimation results to overcome the delay problem of the decisiondirected channel-tracking scheme. Thus, a linear trend predictor is developed to predict one-step ahead channel for improving the performance of data detection in the following subsection.

4.2. Linear Trend Channel Predictor. When the mobile station moves with high velocity and the symbol duration is long, the channel will vary significantly from time $n-1$ to $n$. Since the decision-directed channel-tracking scheme is used, the current channel parameters $\widehat{\boldsymbol{\theta}}_{n}^{\prime}$ are predicted for the current MMSE data detection. The simplest channel predictor is formulated from the previous two estimated channel parameters, $\widehat{\boldsymbol{\theta}}_{n-1}$ and $\hat{\boldsymbol{\theta}}_{n-2}$, with linear extrapolation. An mstep linear trend predictor is formulated as

$$
\widehat{\boldsymbol{\theta}}_{n+m}^{\prime}=\widehat{\boldsymbol{\theta}}_{n}+m \cdot \Delta \hat{\boldsymbol{\theta}}
$$

where

$$
\Delta \widehat{\boldsymbol{\theta}}=\widehat{\boldsymbol{\theta}}_{n}-\widehat{\boldsymbol{\theta}}_{n-1} .
$$

In the case of $m=1$, that is, the one-step linear trend predictor is given by

$$
\widehat{\boldsymbol{\theta}}_{n+1}^{\prime}=\widehat{\boldsymbol{\theta}}_{n}+\Delta \widehat{\boldsymbol{\theta}}_{n}=2 \widehat{\boldsymbol{\theta}}_{n}-\widehat{\boldsymbol{\theta}}_{n-1}
$$

or

$$
\widehat{\boldsymbol{\theta}}_{n}^{\prime}=\widehat{\boldsymbol{\theta}}_{n-1}+\Delta \widehat{\boldsymbol{\theta}}_{n-1}=2 \widehat{\boldsymbol{\theta}}_{n-1}-\widehat{\boldsymbol{\theta}}_{n-2}
$$

where

$$
\begin{aligned}
& \hat{\boldsymbol{\theta}}_{n}^{\prime}=\left[\begin{array}{llllll}
\hat{h}_{n}^{\prime}(0) & \cdots & \hat{h}_{n}^{\prime}(L-1) & \hat{a}_{n}^{\prime}(1) & \cdots & \hat{a}_{n}^{\prime}(P)
\end{array}\right]^{T}, \\
& \hat{h}_{n}^{\prime}(l)=2 \hat{h}_{n-1}(l)-\hat{h}_{n-2}(l), \\
& \hat{a}_{n}^{\prime}(p)=2 \hat{a}_{n-1}(p)-\hat{a}_{n-2}(p) \text {. }
\end{aligned}
$$

Performance of the MMSE detector can be improved with the reliable channel-parameter prediction, and so the onestep parameter prediction in (32) will be used for MMSE detector with a decision-directed channel-tracking scheme in the sequel. 


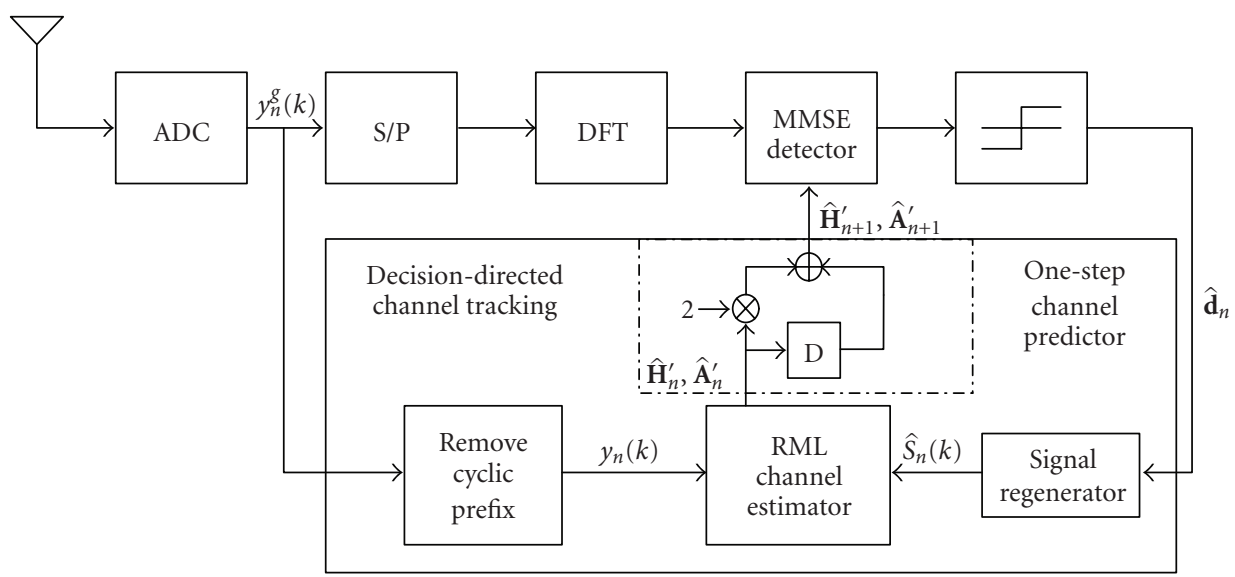

FIGURE 2: Block diagram of the decision-directed channel-tracking algorithm.



FIGURE 3: Symbol-error rate (SER) for different channel-tracking methods in urban area.

4.3. Enhanced MMSE Equalizer for Symbol Detection. A decision-directed channel-tracking algorithm based on the proposed RML method and a simple one-step ahead channel predictor have been developed to overcome the delay effect of the decision-directed scheme under fast fading channel. The MMSE detector using the proposed channel-tracking algorithm is employed now. Before starting the design of MMSE detector, it is necessary to express the received signal $Y_{n}(m)$ in (9) with a matrix form by taking the $N$ consecutive subcarriers for the further development:

$$
\begin{aligned}
\mathbf{Y}_{n} & =\mathbf{H}_{n} \mathbf{S}_{n}+\mathbf{J}_{n} \\
& =\mathbf{H}_{n} \mathbf{C} \mathbf{d}_{n}+\mathbf{A}_{n} \mathbf{U}_{n},
\end{aligned}
$$

where

$$
\begin{aligned}
\mathbf{Y}_{n} & =\left[\begin{array}{llll}
Y_{n}(0) & Y_{n}(1) & \cdots & Y_{n}(N-1)
\end{array}\right]^{T}, \\
\mathbf{d}_{n} & =\left[\begin{array}{llll}
d_{1}(n) & d_{2}(n) & \cdots & d_{N_{u}}(n)
\end{array}\right]^{T}, \\
\mathbf{H}_{n} & =\operatorname{diag}\left\{\left[\begin{array}{llll}
H_{n}(0) & H_{n}(1) & \cdots & H_{n}(N-1)
\end{array}\right]^{T}\right\}, \\
\mathbf{A}_{n} & \left.=\operatorname{diag}\left\{\begin{array}{llll}
A_{n}(0) & A_{n}(1) & \cdots & A_{n}(N-1)
\end{array}\right]^{T}\right\}, \\
\mathbf{U}_{n} & =\left[\begin{array}{llll}
U_{n}(0) & \mathbf{U}_{n}(1) & \cdots & U_{n}(N-1)
\end{array}\right]^{T}, \\
\mathbf{C} & =\left[\begin{array}{llll}
\mathbf{c}_{1} & \mathbf{c}_{2} & \cdots & \mathbf{c}_{N_{u}}
\end{array}\right] .
\end{aligned}
$$

The parameters predicted by the RML-based tracking algorithm in association with the one-step linear trend predictor are used for the MMSE detector. Since the RMLbased tracking algorithm can also estimate the parameters of colored noise, the performance of MMSE detector could be enhanced with this information. In consequence, the proposed method will have better performance than the conventional MMSE detectors with the use of white noise in each subchannel.

To design the MMSE detector $\widehat{\mathbf{w}}_{j, n}$ for the $j$ th user, the following minimum mean-square error detection problem must be solved:

$$
\widehat{\mathbf{w}}_{j, n}=\arg \min _{\mathbf{w}_{j, n}} E\left\{\left|d_{j}(n)-\mathbf{w}_{j, n}^{H} \mathbf{Y}_{n}\right|^{2}\right\}
$$

The solution of optimal detector with consideration of colored noise in (36) is as follows:

$$
\widehat{\mathbf{w}}_{j, n}=\mathbf{R}_{\mathbf{Y Y}}^{-1} \mathbf{r}_{\mathbf{Y} d_{j}},
$$


where

$$
\begin{aligned}
& \mathbf{R}_{\mathbf{Y Y}}=E\left\{\mathbf{Y}_{n} \mathbf{Y}_{n}^{H}\right\} \\
& =\mathbf{H}_{n} \mathbf{C} E\left\{\mathbf{d}_{n} \mathbf{d}_{n}^{H}\right\} \mathbf{C}^{H} \mathbf{H}_{n}^{H}+\mathbf{A}_{n} E\left\{\mathbf{U}_{n} \mathbf{U}_{n}^{H}\right\} \mathbf{A}_{n}^{H} \\
& =\sigma_{d}^{2} \mathbf{H}_{n} \mathbf{C C}^{H} \mathbf{H}_{n}^{H}+N \sigma_{u}^{2} \mathbf{A}_{n} \mathbf{A}_{n}^{H} \\
& =\sigma_{d}^{2} \mathbf{H}_{n} \mathbf{H}_{n}^{H}+N \sigma_{u}^{2} \mathbf{A}_{n} \mathbf{A}_{n}^{H}, \\
& \mathbf{r}_{\mathbf{Y} d_{j}}=E\left\{\mathbf{Y}_{n} d_{j}(n)\right\}=\sigma_{d}^{2} \mathbf{H}_{n} \mathbf{C} \mathbf{e}_{j}, \\
& \mathbf{e}_{j}=\left[\begin{array}{lllllll}
0 & \cdots & 0 & 1 & 0 & \cdots & 0
\end{array}\right]^{T} . \\
& \uparrow \\
& j \text { th }
\end{aligned}
$$

From (37)-(41), the optimal MMSE detector is necessary to know the parameters of $\mathbf{H}_{n}, \mathbf{A}_{n}$, and the variance $\sigma_{u}^{2}$ of the driving noise. These parameters can be obtained from the proposed RML-based parameter-tracking algorithm in (27)(28). However, the current channel parameter is unavailable before the current data detection and the previous estimates are no longer suitable for the current data detection in the fast fading channel. Therefore, the current channel is predicted by feeding the previous two estimates of the RML-based tracking algorithm for the one-step ahead linear trend channel prediction in (31) (see Figure 2). Thus, the parameters fed for the MMSE detection are obtained from the one-step linear trend predictor. By DFT, the parameter prediction is obtained as

$$
\begin{aligned}
& \hat{H}_{n}^{\prime}(m)=\sum_{l=0}^{L-1} \hat{h}_{n}^{\prime}(l) e^{-j 2 \pi l m / N}, \\
& \hat{A}_{n}^{\prime}(m)=\sum_{p=1}^{P} \hat{a}_{n}^{\prime}(p) e^{-j 2 \pi p m / N},
\end{aligned}
$$

where $\hat{h}_{n}^{\prime}(l)$ and $\hat{a}_{n}^{\prime}(p)$ are obtained from the one-step linear trend prediction in (32). Let us denote that

$$
\begin{aligned}
& \hat{\mathbf{H}}_{n}^{\prime}=\operatorname{diag}\left\{\left[\begin{array}{lll}
\hat{H}_{n}^{\prime}(0) & \cdots & \hat{H}_{n}^{\prime}(N-1)
\end{array}\right]\right\}, \\
& \hat{\mathbf{A}}_{n}^{\prime}=\operatorname{diag}\left\{\left[\begin{array}{lll}
\hat{A}_{n}^{\prime}(0) & \cdots & \hat{A}_{n}^{\prime}(N-1)
\end{array}\right]\right\} .
\end{aligned}
$$

Therefore, the MMSE detector in (37) is reformulated as

$$
\widehat{\mathbf{w}}_{j, n}=\left(\sigma_{d}^{2} \hat{\mathbf{H}}_{n}^{\prime} \hat{\mathbf{H}}_{n}^{\prime H}+N \sigma_{u}^{2} \widehat{\mathbf{A}}_{n}^{\prime} \widehat{\mathbf{A}}_{n}^{\prime H}\right)^{-1} \sigma_{d}^{2} \hat{\mathbf{H}}_{n}^{\prime} \mathbf{C e}_{j}
$$

The optimal MMSE detector is obtained by using the proposed RML-based channel-tracking algorithm in association with the one-step linear trend predictor as shown in Figure 2. Finally, the merits of the proposal adaptive decision-directed channel-estimation method are listed in Table 1.

4.4. Performance Analysis for the Proposed Detection Method. Performance of the symbol-detection method is evaluated
TABLE 1: Summarization of the merits of the proposed scheme.

\begin{tabular}{ll}
\hline Item no. & Improvement \\
\hline 1 & $\begin{array}{l}\text { CIR, parameters of the colored noise, and driving } \\
\text { noise estimated simultaneously } \\
\text { Working in the time-domain, reducing } \\
\text { complexity in comparison with the schemes in } \\
\text { frequency-domain } \\
\text { Without pilot symbol, saving bandwidth }\end{array}$ \\
3 & $\begin{array}{l}\text { Including one-step linear trend predictor, useful } \\
\text { for the fast-fading channel }\end{array}$ \\
\hline
\end{tabular}

in this subsection. From (37), the output of the MMSE equalizer for user $j$ is

$$
\begin{aligned}
\bar{d}_{j}(n) & =\widehat{w}_{j, n}^{H} Y_{n} \\
& =\widehat{w}_{j, n}^{H}\left(\mathbf{H}_{n} \mathbf{C} \mathbf{d}_{n}+\mathbf{A}_{n} \mathbf{U}_{n}\right) \\
& =\widehat{w}_{j, n}^{H} \mathbf{H}_{n} \mathbf{C} \mathbf{d}_{n}+\widehat{w}_{j, n}^{H} \mathbf{A}_{n} \mathbf{U}_{n} .
\end{aligned}
$$

Therefore, the average signal-to-noise ratio (SNR) of the output for user $j$ is

$$
\begin{aligned}
\operatorname{SNR}(j, n) & =\frac{E\left\{\widehat{w}_{j, n}^{H} \mathbf{H}_{n} \mathbf{C} \mathbf{d}_{n} \mathbf{d}_{n}^{H} \mathbf{C}^{H} \mathbf{H}_{n}^{H} \widehat{w}_{j, n}\right\}}{E\left\{\widehat{w}_{j, n}^{H} \mathbf{A}_{n} \mathbf{U}_{n} \mathbf{U}_{n}^{H} \mathbf{A}_{n}^{H} \widehat{w}_{j, n}\right\}} \\
& =\frac{\sigma_{d}^{2} E\left\{\widehat{w}_{j, n}^{H} \mathbf{H}_{n} \mathbf{H}_{n}^{H} \widehat{w}_{j, n}\right\}}{N \sigma_{u}^{2} E\left\{\widehat{w}_{j, n}^{H} \mathbf{A}_{n} \mathbf{A}_{n}^{H} \widehat{w}_{j, n}\right\}} .
\end{aligned}
$$

Finally, the decision output of the $n$th symbol for user $j$ is the following:

$$
\hat{d}_{j}(n)=\operatorname{sgn}\left(\bar{d}_{j}(n)\right)
$$

where $\operatorname{sgn}(\cdot)$ denotes the sign function of a decision choice.

For simplicity of analysis, only the case of BPSK will be considered. Assuming that the total interference in (46) can be approximated by a joint Gaussian distribution with zero mean, the symbol detection error probability of user $j$ for the $n$th symbol can be approximated as [31]

$$
P_{e}(j, n)=Q\left(\sqrt{\frac{E\left\{\widehat{w}_{j, n}^{H} \mathbf{H}_{n} \mathbf{H}_{n}^{H} \widehat{w}_{j, n}\right\}}{N \sigma_{u}^{2} E\left\{\widehat{w}_{j, n}^{H} \mathbf{A}_{n} \mathbf{A}_{n}^{H} \widehat{w}_{j, n}\right\}}}\right),
$$

where $Q(x)=(1 / \sqrt{2 \pi}) \int_{x}^{\infty} e^{-\left(t^{2} / 2\right)} d t$ for $x \geq 0$.

\section{Performance Evaluation by Computer Simulation}

Extensive computer simulations are given to demonstrate the performance of the proposed RML channel estimator. Before presenting the simulation results, the parameters of the simulated MC-CDMA systems are described in the following. 
TABle 2: Power delay profile in the typical urban area.

\begin{tabular}{lc}
\hline Delay $(\mu \mathrm{s})$ & Fractional power \\
\hline 0.0 & 0.189 \\
0.2 & 0.379 \\
0.5 & 0.239 \\
1.6 & 0.095 \\
2.3 & 0.061 \\
5.0 & 0.037 \\
\hline
\end{tabular}

5.1. Parameters of MC-CDMA Systems. Table 2 lists the power delay profile in the urban area with the RMS delay $\sigma_{\tau}=1 \mu \mathrm{s}$. If the coherent bandwidth is defined as the bandwidth over which the frequency correlation function is above 0.9 , the coherence bandwidth $B_{c}$ is approximated to $1 / 50 \sigma_{\tau}$ [32]. The central frequency $f_{c}$ is $1.8 \mathrm{GHz}$ in the MCCDMA system. The total bandwidth $B W$ is $1.024 \mathrm{MHz}$ which is divided into 512 subchannels. The subchannel spacing is then $\Delta f=2 \mathrm{kHz}$. An additional $8 \mu \mathrm{s}$ guard interval duration is used to provide protection from ISI due to channel multipath delay spread. The length of the adopted Walsh-Hadamard code is $N=64$ chips. Thus, the MCCDMA system can support the maximum number of active users $N_{u}=64$. It is also assumed that the channel remains approximately constant during one MC symbol period. In the following simulations, the data modulation scheme is QPSK.

5.2. Simulation Results. The following examples are simulated for 50 runs, and each run with 2000 MC-CDMA symbols. The number of active user is 32 . The forgetting factor $\lambda$ is 0.995 . The initial value of $P_{k, n}$ is a unitary matrix. In all cases, we normalize the gain of delay paths so that

$$
\sum_{l=0}^{L-1} E\left\{\left|h_{n}(l)\right|^{2}\right\}=1
$$

Performance of the proposed method is compared with other methods in several situations.

5.2.1. Slow Velocity. The velocity is $10 \mathrm{~km} / \mathrm{hr}$ (the fading rate $\left.f_{d} T=0.0086\right)$ in this simulation. The proposed method without one-step predictor is used in this situation. The symbol-error rate (SER) versus signal-to-noise ratio (SNR) is illustrated in Figure 3. It can be seen that the SER of the MMSE detector with perfect channel estimation is a lower bound. In the $2 \mathrm{D}$ pilot-symbol-aided channel estimation [12], the spaces between pilots in the time domain are chosen according to coherence time $T_{c} \approx 9 / 16 \pi f_{d}$ [32] if the coherent time is defined as the time over which the correlation function is above 0.5 . The spaces between pilots in the frequency domain are chosen based on the coherence bandwidth $B_{c}$. A frequency-domain channel estimator introduced in [18] is Kalman filter with a decision-directed scheme. Since the frequency-domain channel estimator has no immunity to colored noise, its performance is the worst

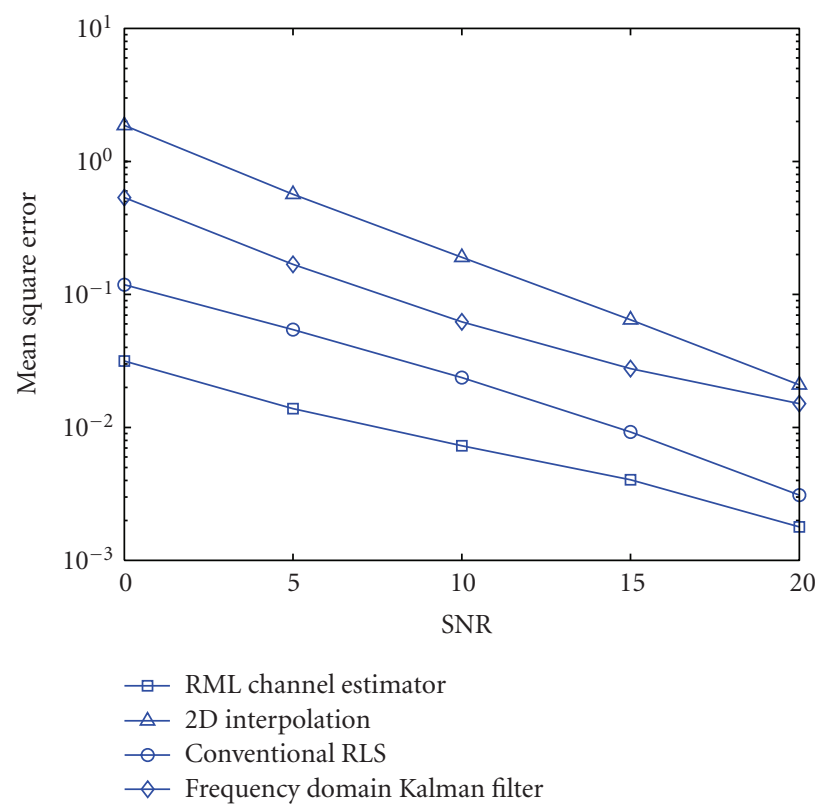

FIGURE 4: MSE of different channel-tracking methods as $v=$ $10 \mathrm{~km} / \mathrm{hr}\left(f_{d} T=0.0086\right)$ in urban area.

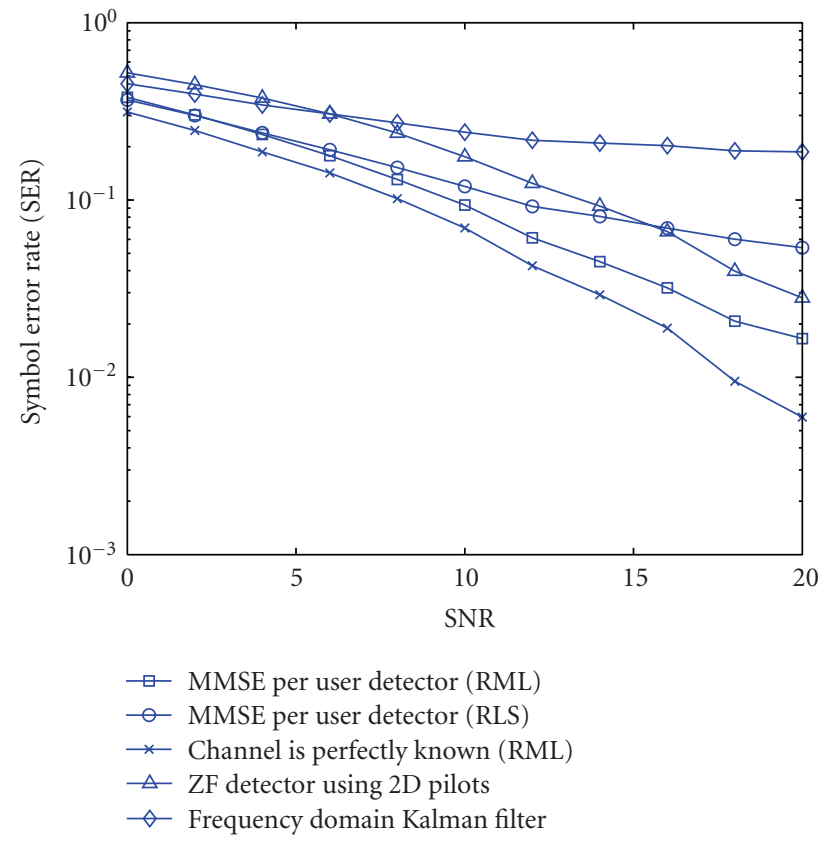

FIGURE 5: Symbol-error rate (SER) for different channel-tracking methods as $v=60 \mathrm{~km} / \mathrm{hr}\left(f_{d} T=0.0516\right)$ in urban area.

of the four methods. The conventional RLS can estimate the channel parameters but not the parameters of the colored noise. Therefore, it cannot achieve the MMSE detector and its performance is worse than the proposed method. The mean-square error (MSE) versus SNR is presented in Figure 4. The proposed RML estimator has the lowest MSE, and so its performance shown in Figure 3 is very close to the performance with the perfectly known channel. 


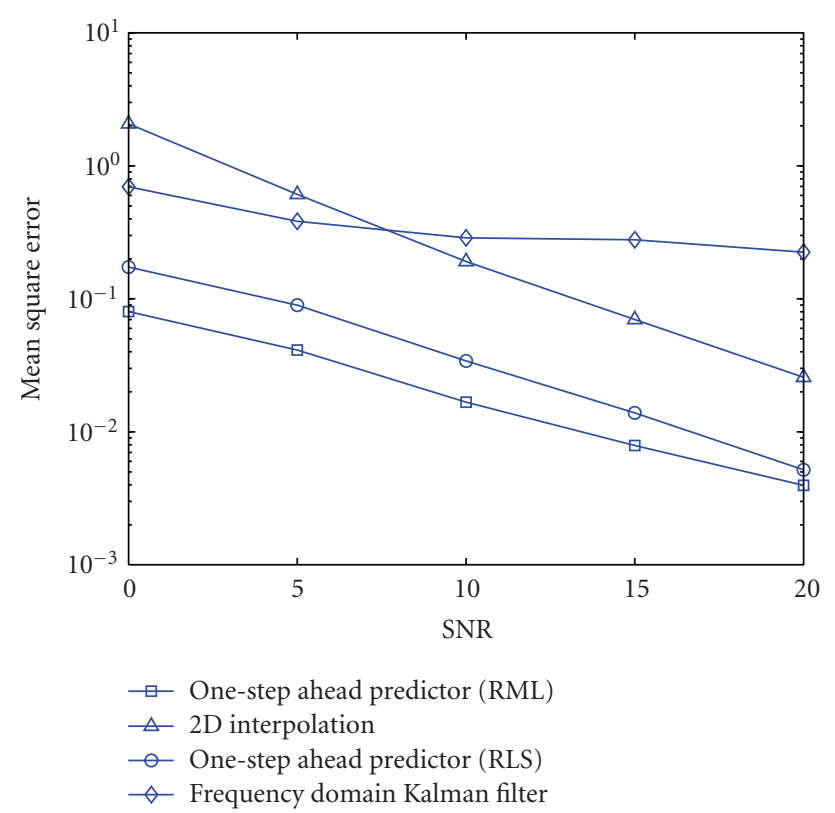

FIgURE 6: MSE of different channel-tracking methods as $v=$ $60 \mathrm{~km} / \mathrm{hr}\left(f_{d} T=0.0516\right)$ in urban area.

5.2.2. High Velocity. When the mobile station moves with high velocity, the channel will vary significantly in a symbol duration. The conventional decision-directed methods will fail in this situation because all of them feed the previous estimates to the MMSE detector for current detection. Based on the decision-directed RML-based channel-tracking algorithm, we further design a one-step channel predictor in Section 4 to overcome the delay problem. The SER and MSE of channel tracking using different methods with and without a one-step predictor are compared in Figures 5 and 6 , in which the velocity $60 \mathrm{~km} / \mathrm{hr}\left(f_{d} T=0.0516\right)$ is considered for the design procedure. It is obvious that the proposed RML-based tracking method in association with a one-step channel predictor has the most accurate prediction of the current channel for current data detection when the channel is fast fading. Therefore, the SER of the proposed method is the lowest. When the SNR is below $8 \mathrm{~dB}$, the MSE and SER of the frequency-domain Kalman filter are worst because it feeds the previous estimates to the MMSE detector.

5.2.3. Different Velocities with Fixed SNR. The performances of different estimation methods are compared when the mobile station moves at different velocities with SNR = $10 \mathrm{~dB}$, as shown in Figure 7. The proposed method without one-step predictor has the best performance with slow velocity because the channel variation is slow. However, when the mobile moves with high velocity, the proposed method with a one-step channel predictor has the most accurate prediction of current channel for the current MMSE detection.

From these simulation results, the MMSE detector with the proposed time-domain RML-based channel-tracking

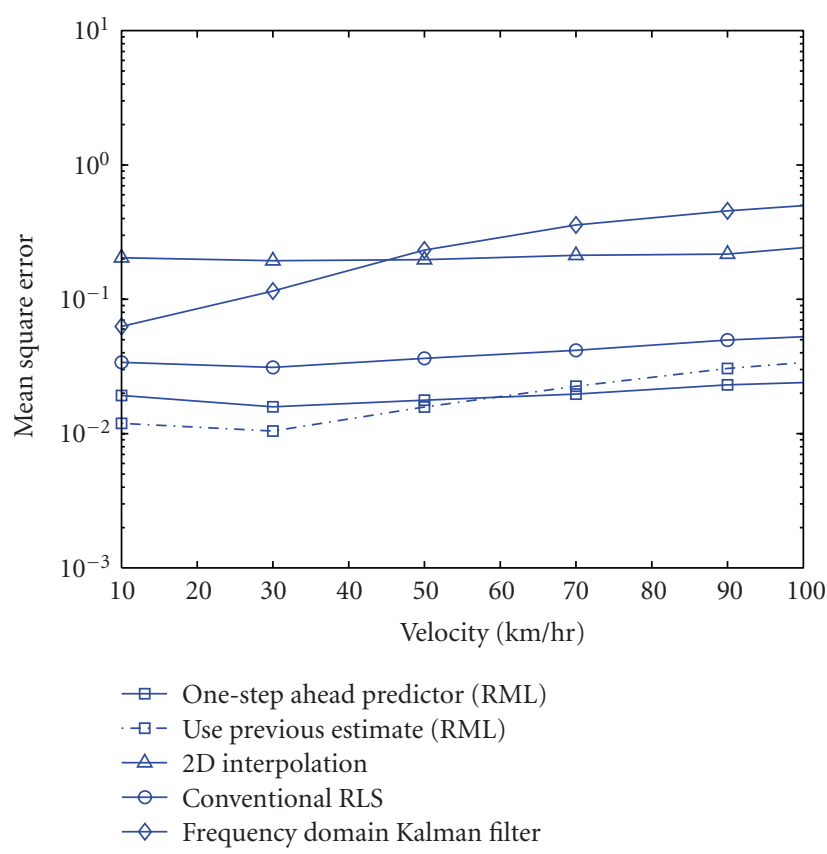

FIGURE 7: MSE of channel tracking versus speed of user.

algorithm and the one-step channel prediction can significantly improve the performance of MC-CDMA under the fast fading channel and colored noise. They are useful for practical applications in the MC-CDMA communication systems.

\section{Conclusions}

This work has presented a channel-estimation method and an enhanced MMSE detector for an MC-CDMA system under the rapidly fading channel and colored noise. The proposed RML method can simultaneously estimate the parameters of channel and colored noise, and the variance of driving noise in the time domain with a simple scheme. The MMSE detector will be enhanced by using the estimated parameters. The proposed channel-estimation method can work in the time domain because the parameters of colored noise are estimated by using the residues generated in the process of the recursive channel-estimation algorithm. Another advantage is that the number of estimated parameters in the time domain is less than that in the frequency domain. Thus, the computational complexity can be significantly reduced by decreasing the number of estimated parameters. The decision-directed RML-based channel-tracking algorithm can reduce occupancy of the available bandwidth. Furthermore, a one-step linear trend channel predictor is proposed to feed more accurate channel parameters to MMSE detector for improving the current data detection. The MSE of the proposed channel-estimation scheme is improved and the SER is very close to the case with the perfectly known channel parameters. The proposed scheme provides better performance than the other methods for MC-CDMA systems under the rapidly fading channel 
and colored noise from simulation. It is because none of the conventional channel-estimation methods can estimate the parameters of colored noise in real time or they have other drawbacks, that is, occupying of large bandwidth, inaccuracy in the fast fading channel and under the colored noise, and so forth. Therefore, the proposed decision-directed RMLbased channel-tracking method with a one-step linear trend channel predictor is very useful for MC-CDMA systems over rapidly Rayleigh fading channel with colored noise.

\section{References}

[1] A. J. Viterbi, Principle of Spread Spectrum Communications, Addison-Wesley, Reading, Mass, USA, 1995.

[2] K. Tachikawa, W-CDMA Mobile Communications Systems, John Wiley \& Sons, New York, NY, USA, 2002.

[3] E. Dahlman, B. Gudmundson, M. Nilsson, and J. Sköld, "UMTS/IMT-2000 based on wideband CDMA," IEEE Communications Magazine, vol. 36, no. 9, pp. 70-80, 1998.

[4] R. van Nee, G. Awater, M. Morikura, H. Takanashi, M. Webster, and K. W. Halford, "New high-rate wireless LAN standards," IEEE Communications Magazine, vol. 37, no. 12, pp. 82-88, 1999.

[5] IEEE Std. 802.16a, "Local and metropolitan area networkspart 16, air interface for fixed broadband wireless access systems," 2003.

[6] N. Yee and J. P. Linnartz, "Wiener filtering of multi-carrier CDMA in Rayleigh fading channel," in Proceedings of the 5th IEEE International Symposium on Personal, Indoor and Mobile Radio Communications (PIMRC '94), vol. 4, pp. 1344-1347, The Hague, The Netherlands, September 1994.

[7] H. Xing and M. Renfors, "Performance evaluation of the multi-carrier CDMA system with frequency domain equalization," in Proceedings of the IEEE Vehicular Technology Conference (VTC '99), pp. 2362-2366, Amsterdam, The Netherlands, September 1999.

[8] A. B. Djebbar, K. Abed-Meraim, and A. Djebbari, "Blind and semi-blind equalization of downlink MC-CDMA system exploiting guard interval redundancy and excess codes," IEEE Transactions on Communications, vol. 57, no. 1, pp. 156-163, 2009.

[9] T. Mueller, H. Rohling, and R. Gruenheid, "Comparison of different detection algorithms for OFDM-CDMA in broadband Rayleigh fading," in Proceedings of the 45th IEEE Vehicular Technology Conference (VTC '95), pp. 835-838, Chicago, Ill, USA, July 1995.

[10] S. Kaiser, "Analytical performance evaluation of OFDMCDMA mobile radio systems," in Proceedings of the 1st European Personal and Mobile Communications Conference (EPMCC '95), pp. 215-220, Bologna, Italy, November 1995.

[11] D. N. Kalofonos and J. G. Proakis, "Performance of the multistage detector for a MC-CDMA system in a Rayleigh fading channel," in Proceedings of IEEE Global Telecommunicatins Conference (GLOBECOM '93), vol. 3, pp. 1784-1788, Houston, Tex, USA, November-December 1993.

[12] S. Kaiser and P. Hoeher, "Performance of multi-carrier CDMA systems with channel estimation in two dimensions," in Proceedings of the 8th IEEE International Symposium on Personal, Indoor and Mobile Radio Communications (PIMRC '97), pp. 115-119, Helsinki, Finland, September 1997.

[13] P. Hoeher, S. Kaiser, and P. Robertson, "Two-dimensional pilot-symbol-aided channel estimation by Wiener filtering," in
Proceedings of the IEEE International Conference on Acoustics, Speech, and Signal Processing (ICASSP '97), pp. 1845-1848, Munich, Germany, April 1997.

[14] A. Nagate and T. Fujii, "A study on channel estimation methods for time-domain spreading MC-CDMA systems," IEEE Transactions on Wireless Communications, vol. 7, no. 12, pp. 5233-5237, 2008.

[15] S. Cacopardi, F. Frescura, F. Gatti, and G. Reali, "Channel estimation and tracking of an indoor orthogonal multicarrier DS-CDMA system using measured channel delay profiles," in Proceedings of the IEEE Vehicular Technology Conference (VTC '96), pp. 1559-1563, Atlanta, Ga, USA, May 1996.

[16] S. Cacopardi, F. Frescura, and G. Reali, "Performance comparison of multicarrier DS-SS radio access schemes for WLAN using measured channel delay profiles," in Proceedings of the 47th IEEE Vehicular Technology Conference (VTC '97), pp. 1877-1881, Phoenix, Ariz, USA, May 1997.

[17] B.-S. Chen and J.-F. Liao, "Adaptive MC-CDMA multiple channel estimation and tracking over time-varying multipath fading channels," IEEE Transactions on Wireless Communications, vol. 6, no. 6, pp. 2328-2337, 2007.

[18] D. N. Kalofonos, M. Stojanovic, and J. G. Proakis, "Performance of adaptive MC-CDMA detectors in rapidly fading rayleigh channels," IEEE Transactions on Wireless Communications, vol. 2, no. 2, pp. 229-239, 2003.

[19] J. Wang and H. Huang, "MC DS/SFH-CDMA systems for overlay systems," IEEE Transactions on Wireless Communications, vol. 1, no. 3, pp. 448-455, 2002.

[20] S. Kondo and L. B. Milstein, "Performance of mMulticarrier DS CDNA systems," IEEE Transactions on Communications, vol. 44, no. 2, pp. 238-246, 1996.

[21] S. Akkarakaran and P. P. Vaidyanathan, "Results on principal component filter banks: colored noise suppression and existence issues," IEEE Transactions on Information Theory, vol. 47, no. 3, pp. 1003-1020, 2001.

[22] M. Sternad and A. Ahlen, "The structure and design of realizable decision feedback equalizers for IIR channels with colored noise," IEEE Transactions on Information Theory, vol. 36, no. 4, pp. 848-858, 1990.

[23] Y. Wang and L. B. Milstein, "Rejection of multiple narrowband interference in both BPSK and QPSK DS spreadspectrum systems," IEEE Transactions on Communications, vol. 36, no. 2, pp. 195-204, 1988.

[24] L. A. Rusch and H. V. Poor, "Narrowband interference suppression in CDMA spread spectrum communications," IEEE Transactions on Communications, vol. 42, no. 2, pp. 19691979, 1994.

[25] P.-R. Chang and J.-T. Hu, "Narrow-band interference suppression in spread-spectrum CDMA communications using pipelined recurrent neural networks," IEEE Transactions on Vehicular Technology, vol. 48, no. 2, pp. 467-477, 1999.

[26] W. Mao, H. Tsao, and F. Chang, "Anti-jamming solution of GPS receiver using nonlinear adaptive predictor," in Proceedings of the14thInternational Technical Meeting of the Satellite Division of the Institute of Navigation (ION GPS '01), pp. 970974, Salt Lake City, Utah, USA, September 2001.

[27] H. Vincent Poor, "Active interference suppression in CDMA overlay systems," IEEE Journal on Selected Areas in Communications, vol. 19, no. 1, pp. 4-20, 2001.

[28] R. Johansson, System Modeling and Identification, PrenticeHall International, Englewood Cliffs, NJ, USA, 1993.

[29] J. E. Kim, S. H. Yoon, S. J. Kang, and C. E. Kang, "Multi-carrier CDMA system using a code orthogonalising filter," Electronics Letters, vol. 34, no. 16, pp. 1557-1558, 1998. 
[30] B.-K. Lee and B.-S. Chen, "Identification of fuzzy T-S ARMAX models," in Proceedings of the IEEE International Conference on Fuzzy Systems, pp. 1019-1024, Budapest, Hungary, July 2004.

[31] J. G. Proakis, Digital Communications, McGraw-Hill, New York, NY, USA, 4th edition, 2000.

[32] T. S. Rappaport, Wireless Communications: Principles and Practice, Prentice Hall PTR, Upper Saddle River, NJ, USA, 2002. 